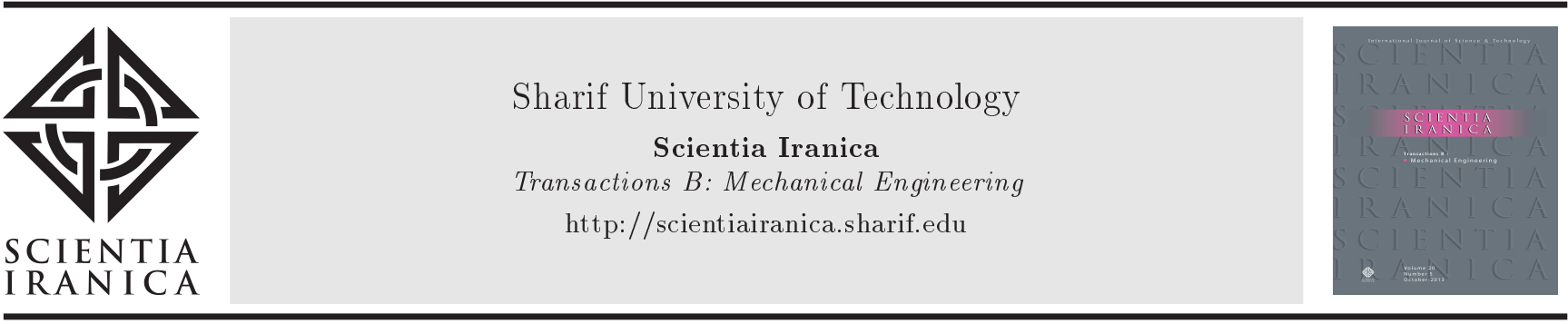

\title{
The impact of compliant walls on magneto hydrodynamics peristalsis of Jeffrey material in a curved configuration
}

 \\ a. Department of Mathematics, Quaid-i-Azam University 45320, Islamabad 44000, Pakistan. \\ b. Department of Mathematics, Faculty of Science, King Abdulaziz University, Jeddah 21589, Saudi Arabia. \\ Received 23 July 2016; received in revised form 9 December 2016; accepted 28 August 2017
}

\author{
KEYWORDS \\ Jeffrey fluid; \\ Radial magnetic field; \\ Compliant wall \\ conditions; \\ Soret and dufour \\ effects; \\ Curved channel.
}

\begin{abstract}
The primary aim of the current attempt is to analyze the peristaltic flow of non-Newtonian material in a curved channel subject to two salient features, namely the Soret and Dufour and radial magnetic field. Channel walls are of compliant characteristics. The problem formulations for constitutive equations of Jeffrey fluid are made. The lubrication approach is implemented to simplify the mathematical analysis. Dimensionless problems of stream function, temperature, and concentration are computed numerically. Characteristics of distinct variables on the velocity, temperature, coefficient of heat transfer, and concentration are examined. Besides, the graphical results indicate that the velocity profile enhances the compliant wall parameters significantly, primarily due to the resistance characteristics of Lorentz force velocity profile decays. Furthermore, it is noted that the temperature profile enhances larger Dufour number; however, reverse behavior is noticed in the concentration profile when Soret and Schmidt numbers are increased.
\end{abstract}

(C) 2018 Sharif University of Technology. All rights reserved.

\section{Introduction}

The peristalsis regarding flows of viscous and inviscid materials has attracted the attention of recent researchers in view of their physiological and engineering applications. Physiological processes involve peristalsis consisting of urine movement through kidney to bladder, chyme transportation in intestine, spermatozoa transport, food swallowing via esophagus, and vasomotion in tiny blood vessels. Peristalsis is also quite prevalent in heart-lung machine, roller and finger pumps, sanitary and toxic liquid transport, and food, paper,

*. Corresponding author. Tel.: + 92 5190642172 ;

E-mail address: farooq.fmg89@yahoo.com (S. Farooq)

doi: $10.24200 /$ sci. 2017.4322 and cosmetic industries. Pioneering research studies on peristalsis involving viscous materials were theoretically and experimentally analyzed by Latham [1] and Shapiro et al. [2]. Afterwards, ample investigations have been conducted into peristalsis subject and the diverse aspects of rheological characteristics, heat and mass transfer, magneto hydrodynamics, wave shapes, geometries, etc. (see [3-21] and many studies therein). Much importance in the past was directed to the peristalsis of Newtonian and non-Newtonian liquids in a straight channel which seems not realistic in several applications relevant to physiological and engineering processes. In addition, the Magneto Hydro Dynamic (MHD) effect, in the existing attempts, is mostly through the consideration of applied constant magnetic field. However, there is no doubt that MHD flows are of great interest in the MHD power generators, pumps, 
accelerators, hyperthermia, bleeding minimization during surgeries, treatment of cancer tumor, blockage treatment in the arteries, magnetic endoscopy, Magnetic Resonance Imaging (MRI), purification of molten metals from non-metallic inclusions, and several others. Keeping in mind the above-mentioned uses of Magneto Hydro Dynamic (MHD), Ellahi et al. [22] reported Ion slip and Hall aspects in MHD peristalsis of Jeffrey fluid in an irregular rectangular duct. In addition, Hayat et al. [23] described the MHD peristalsis in Jeffrey material through curved channel with convective constraints. Few recent developments in MHD peristaltic flow can be seen through [24-27]. Hence, the objective of the present communication is to predict the influence of radial magnetic field on the peristalsis of Jeffrey material in a channel. Plus, the effect of curvature is studied. Moreover, the compliant wall properties of curved channel are analyzed. Heat and mass transfer are examined in the presence of Dufour and Soret phenomenon. Dimensionless problems subject to lubrication approach are studied. The outcome in discussion section consists of salient features of sundry parameters entering into the formulation. This work is structured as follows.

The first section contains introduction. Sections 2 and 3 consist of formulation and technique for the solutions. Section 4 discusses various pertinent variables. Conclusions are given in Section 5 .

\section{Problems formulation}

In Figure 1, we consider the peristaltic flow of an incompressible Jeffrey liquid in curved channel of a width of $2 a_{0}$, twisted in a circle of radius, $R^{*}$, and a center at $O$. We consider $\overline{v_{1}}$ as the velocity along radial $(\bar{r})$ and $\bar{v}_{2}$ as the velocity along axial $(\bar{x})$ directions. A radially imposed magnetic field of strength, $B_{0}$, is applied along radial direction. In addition, heat and mass transfer is studied. Mathematical analysis also involves the Soret and Dufour effects.

The channel walls' temperatures are denoted by $T_{0}$ and $T_{1}$. The concentrations at the walls are $C_{0}$

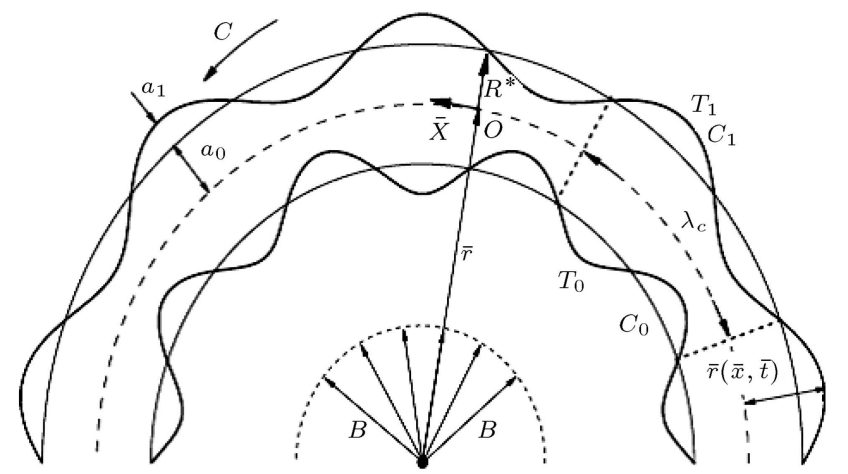

Figure 1. Physical diagram. and $C_{1}$. The fluid flow here is due to propagation of the channel walls. Mathematical description of wall surfaces is as follows:

$$
\bar{h}(\bar{x}, \bar{t})=a_{0}+a_{1} \sin \left(\frac{2 \pi}{\lambda_{c}}(\bar{x}-c \bar{t})\right) .
$$

Here, $c$ represents the wave speed, $a_{0}$ the half width of the channel, $a_{1}$ the wave amplitude, $\lambda_{c}$ the wavelength, and $\bar{t}$ the time in fixed frame. Velocity, $\overline{\mathbf{V}}$, can be defined as follows:

$$
\overline{\mathbf{V}}=\left(\bar{v}_{1}(\bar{x}, \bar{r}, \bar{t}), \bar{v}_{2}(\bar{x}, \bar{r}, \bar{t}), 0\right) .
$$

extra stress tensor $\overline{\mathbf{S}}$ for Jeffrey fluid model is given by $[9,10,20-23,28]$ :

$$
\overline{\mathbf{S}}=\frac{\mu}{1+\lambda_{1}}\left(\overline{\mathbf{A}}_{1}+\lambda_{2} \frac{d}{d \bar{t}} \overline{\mathbf{A}}_{1}\right)
$$

In the above relation, $\mu, \lambda_{1}$, and $\lambda_{2}$ denote dynamic viscosity, ratio of relaxation to retardation time, and retardation time, respectively. The present study can be reduced to viscous material when $\lambda_{1}=\lambda_{2}=0$. Moreover:

$$
\begin{aligned}
& \overline{\mathbf{A}}_{1}=(\operatorname{grad} \overline{\mathbf{V}})+(\operatorname{grad} \overline{\mathbf{V}})^{*} \\
& \frac{d}{d \bar{t}} \overline{\mathbf{A}}_{1}=\frac{\partial}{\partial \bar{t}}\left(\overline{\mathbf{A}}_{1}\right)+(\overline{\mathbf{V}} \cdot \nabla) \overline{\mathbf{A}}_{1} .
\end{aligned}
$$

The flow under consideration is governed by the following expressions:

$$
\begin{aligned}
& \frac{\partial}{\partial \bar{r}}\left\{\left(R^{*}+\bar{r}\right) \bar{v}_{1}\right\}+R^{*} \frac{\partial \bar{v}_{2}}{\partial \bar{x}}=0 \\
& \rho\left[\frac{\partial \bar{v}_{1}}{\partial \bar{t}}+\bar{v}_{1} \frac{\partial \bar{v}_{1}}{\partial \bar{r}}+\frac{R^{*} \bar{v}_{2}}{\bar{r}+R^{*}} \frac{\partial \bar{v}_{1}}{\partial \bar{x}}-\frac{\bar{v}_{2}^{2}}{\bar{r}+R^{*}}\right]= \\
& \quad-\frac{\partial \bar{p}}{\partial \bar{r}}+\frac{1}{R^{*}+\bar{r}} \frac{\partial}{\partial \bar{r}}\left\{\left(\bar{r}+R^{*}\right) \bar{S}_{\bar{r} r}\right\} \\
& +\left(\frac{R^{*}}{\bar{r}+R^{*}}\right) \frac{\partial \bar{S}_{\overline{r x}}}{\partial \bar{x}}-\frac{\bar{S}_{\overline{x x}}}{\bar{r}+R^{*}}, \\
& \left.\rho \frac{\partial \bar{v}_{2}}{\partial \bar{t}}+\bar{v}_{1} \frac{\partial \bar{v}}{\partial \bar{r}}+\frac{R^{*} \bar{v}_{2}}{\bar{r}+R^{*}} \frac{\partial \bar{v}}{\partial \bar{x}}-\frac{\bar{v}_{1} \bar{v}_{2}}{\bar{r}+R^{*}}\right]= \\
& -\left(\frac{R^{*}}{\bar{r}+R^{*}}\right) \frac{\partial \bar{p}}{\partial \bar{x}}+\frac{1}{\left(\bar{r}+R^{*}\right)^{2}} \frac{\partial}{\partial \bar{r}}\left\{\left(\bar{r}+R^{*}\right)^{2} \bar{S}_{\overline{r x}}\right\} \\
& +\left(\frac{R^{*}}{\bar{r}+R^{*}}\right) \frac{\partial \bar{S}_{\overline{x x}}}{\partial \bar{x}}-\frac{\sigma B_{0}^{2}}{\bar{r}+R^{*}} \bar{v}_{2},
\end{aligned}
$$




$$
\begin{aligned}
& \rho c_{p}\left[\frac{\partial T}{\partial \bar{t}}+\bar{v}_{1} \frac{\partial T}{\partial \bar{r}}+\frac{\bar{v}_{2} R^{*}}{\bar{r}+R^{*}} \frac{\partial T}{\partial \bar{x}}\right] \\
& =\kappa\left[\frac{\partial^{2} T}{\partial \bar{r}^{2}}+\frac{1}{R^{*}+\bar{r}} \frac{\partial T}{\partial \bar{r}}+\frac{R^{* 2}}{\left(\bar{r}+R^{*}\right)^{2}} \frac{\partial^{2} T}{\partial \bar{x}^{2}}\right] \\
& +\mu\left[\left(\frac{\partial \bar{v}_{2}}{\partial \bar{r}}-\frac{\bar{v}_{2}}{\bar{r}+R^{*}}+\frac{R^{*}}{\bar{r}+R^{*}} \frac{\partial \bar{v}_{1}}{\partial \bar{x}}\right)\right. \\
& \left.S_{\overline{r x}}+\frac{\partial \bar{v}_{2}}{\partial \bar{r}}\left(S_{\overline{r r}}-S_{\overline{x x}}\right)\right] \\
& +\frac{D K_{T}}{C_{s}}\left[\frac{\partial^{2}}{\partial \bar{r}^{2}}+\frac{1}{\bar{r}+R^{*}} \frac{\partial}{\partial \bar{r}}+\frac{R^{* 2}}{\left(\bar{r}+R^{*}\right)^{2}} \frac{\partial^{2}}{\partial \bar{x}^{2}}\right] C \\
& {\left[\frac{\partial}{\partial \bar{t}}+\bar{v}_{1} \frac{\partial}{\partial \bar{r}}+\frac{\bar{v}_{2} R^{*}}{\bar{r}+R^{*}} \frac{\partial}{\partial \bar{x}}\right] C} \\
& =D\left[\frac{\partial^{2}}{\partial \bar{r}^{2}}+\frac{1}{\bar{r}+R^{*}} \frac{\partial}{\partial \bar{r}}+\frac{R^{* 2}}{\left(\bar{r}+R^{*}\right)^{2}} \frac{\partial^{2}}{\partial \bar{x}^{2}}\right] C \\
& +\frac{D K_{T}}{T_{m}}\left[\frac{\partial^{2}}{\partial \bar{r}^{2}}+\frac{1}{\bar{r}+R^{*}} \frac{\partial}{\partial \bar{r}}+\frac{R^{* 2}}{\left(\bar{r}+R^{*}\right)^{2}} \frac{\partial^{2}}{\partial \bar{x}^{2}}\right]_{(10}
\end{aligned}
$$

In the above equations, $\bar{p}$ denotes the pressure, $\rho$ the fluid density, $\kappa$ the thermal conductivity, $c_{p}$ the specific heat, $\bar{t}$ the time, $\bar{S}_{\overline{x x}}, \bar{S}_{\overline{r r}}, \bar{S}_{\overline{r x}}$, the stress components, $T$ and $C$ the temperature and concentration of the fluid, respectively. The subjected boundary conditions are:

$$
\begin{aligned}
\bar{v}_{2}= & 0 \quad \text { at } \quad \bar{r}= \pm \bar{h} \\
{[-} & \left.\tau \frac{\partial^{3}}{\partial \bar{x}^{3}}+m \frac{\partial^{3}}{\partial \bar{x} \partial \bar{t}^{2}}+d \frac{\partial^{2}}{\partial \bar{t} \partial \bar{x}}\right] \bar{h} \\
= & \frac{1}{R^{*}\left(\bar{r}+R^{*}\right)} \frac{\partial}{\partial \bar{r}}\left\{\left(\bar{r}+R^{*}\right)^{2} S_{\overline{r x}}\right\} \\
& +\frac{\partial S_{\overline{x x}}}{\partial \bar{x}}-\rho\left(\bar{r}+R^{*}\right) \\
& \times\left[\frac{\partial \bar{v}_{2}}{\partial \bar{t}}+\bar{v}_{1} \frac{\partial \bar{v}_{2}}{\partial \bar{r}}+\frac{R^{*} \bar{v}_{2}}{\bar{r}+R^{*}} \frac{\partial \bar{v}_{2}}{\partial \bar{x}}-\frac{\bar{v}_{1} \bar{v}_{2}}{R^{*}+\bar{r}}\right] \\
& -\frac{H a^{2} \bar{v}_{2}}{R^{*}\left(\bar{r}+R^{*}\right)} \quad \text { at } \quad \bar{r}= \pm \bar{h},
\end{aligned}
$$

$$
\begin{aligned}
& T=T_{1}, \quad T=T_{0} \quad \text { at } \quad \bar{r}= \pm \bar{h} \quad \text { and } \\
& C=C_{1}, \quad C=C_{0} \quad \text { at } \quad \bar{r}= \pm \bar{h} .
\end{aligned}
$$

We denote the dimensionless parameters as follows:

$$
\begin{aligned}
& x=\frac{2 \pi \bar{x}}{\lambda_{c}}, \quad r=\frac{\bar{r}}{a_{0}}, \quad v_{1}=\frac{\bar{v}_{1}}{c}, \quad v_{2}=\frac{\bar{v}_{2}}{c}, \\
& \delta=\frac{2 \pi a_{0}}{\lambda_{c}}, \quad \eta= \pm \frac{\bar{h}}{a_{0}}, \quad p=\frac{2 \pi a_{0}^{2} \bar{p}}{c \mu \lambda_{c}}, \\
& \mathrm{Ha}=\left(\frac{\sigma}{\mu}\right)^{1 / 2} B_{0} a_{0}, \quad \operatorname{Re}=\frac{\rho c a_{0}}{\mu}, \quad k=\frac{R^{*}}{a_{0}}, \\
& \operatorname{Pr}=\frac{\mu c_{p}}{\kappa}, \quad t=\frac{c \bar{t}}{\lambda_{c}}, \quad \theta=\frac{T-T_{0}}{T_{1}-T_{0}}, \\
& \phi=\frac{C-C_{0}}{C_{1}-C_{0}}, \quad \mathrm{Ec}=\frac{c^{2}}{\left(T_{0}-T_{1}\right) c_{p}}, \quad \mathrm{Br}=\operatorname{Pr} \mathrm{Ec}, \\
& \mathrm{Sc}=\frac{\mu}{\rho D}, \quad \mathrm{Sr}=\frac{\rho D K_{T}\left(T_{1}-T_{0}\right)}{\mu\left(C_{1}-C_{0}\right)}, \\
& \mathrm{Du}=\frac{D K_{T}\left(C_{1}-C_{0}\right)}{C_{s} \mu c_{p}\left(T_{1}-T_{0}\right)}, \quad E_{1}=\frac{-\tau a_{0}^{3}}{\lambda_{c}^{3} \mu c}, \\
& E_{2}=\frac{m c a_{0}^{3}}{\lambda_{c}^{3} \mu}, \quad E_{3}=\frac{d a_{0}^{3}}{\lambda_{c}^{3} \mu} .
\end{aligned}
$$

Here, $k$ represents the curvature parameter, $\delta$ the wave, Ha the Hartman, Re the Reynolds, Pr the Prandtl, Ec the Eckert, Br the Brinkman, Du the Dufour, Sr the Soret, Sc the Schmidt as dimensionless numbers, and $E_{1}, E_{2}$, and $E_{3}$ the wall tension, mass characterizing, and wall damping parameters, respectively.

The dimensionless representation of the boundary conditions (11)-(13) yields:

$$
\begin{gathered}
v_{2}=0 \text { at } r= \pm \eta \\
{\left[E_{1} \frac{\partial^{3}}{\partial x^{3}}+E_{2} \frac{\partial^{3}}{\partial x \partial t^{2}}+E_{3} \frac{\partial^{2}}{\partial t \partial x}\right] \eta} \\
=\frac{1}{k(r+k)} \frac{\partial}{\partial r}\left\{(r+k)^{2} S_{r x}\right\}+\frac{\delta}{k} \frac{\partial S_{x x}}{\partial x} \\
-\frac{\operatorname{Re}(r+k)}{k} \times\left[\delta \frac{\partial v_{2}}{\partial t}+v_{1} \frac{\partial v_{2}}{\partial r}\right. \\
\left.+\frac{k \delta v_{2}}{k+r} \frac{\partial v_{2}}{\partial x}-\frac{v_{1} v_{2}}{k+r}\right]-\frac{\mathrm{Ha}^{2} v_{2}}{k(r+k)} \\
\theta=1, \quad \theta=0, \quad \text { at } \quad r= \pm \eta \quad \text { and } \\
\phi=1, \quad \phi=0 \quad \text { at } \quad r= \pm \eta,
\end{gathered}
$$

where boundary condition in Eq. (15) represents the no-slip conditions at walls, and condition for the compliant wall at upper and lower walls is given in Eq. (16), 
respectively. Boundary condition for temperature and concentration profiles is represented in Eq. (17).

Velocity components in terms of stream function, $\psi$, can be defined by:

$$
v_{1}=\delta \frac{k}{r+k} \psi_{x}, \quad \nu_{2}=-\psi_{r}
$$

In large peristaltic wavelength, low Reynolds number approximations play a vital role (see $[2,12,29,30])$. The existence of such assumptions in physiology is justified through the transportation of chyme in small intestine [31]. In such conditions, the wavelength $\left(\lambda_{c}=8.01\right)$ of peristaltic wave is very large in comparison to the halfwidth $\left(a_{0}=1.25\right)$ of the channel/tube i.e. $\left(a_{0} / \lambda_{c}=\right.$ 0.156). Further, Lew et al. [32] examined liquids in small intestine with low Reynolds number. Urine transport in the human ureter is also the application of low Reynolds number approximation. In view of the above-mentioned applications of long wavelength and low Reynolds number assumption in peristalsis, Eq. (6) is satisfied identically and Eqs. (7)-(10) lead to the following expressions:

$$
\begin{aligned}
& \frac{d p}{d r}=0 \\
& \frac{k}{r+k} \frac{d p}{d x}=\frac{1}{(r+k)^{2}} \frac{\partial}{\partial r}\left\{(r+k)^{2} S_{r x}\right\} \\
& \quad-\frac{\mathrm{Ha}^{2}}{(r+k)^{2}}\left(\psi_{r}\right), \\
& \theta_{r r}+\frac{1}{r+k} \theta_{r}+\operatorname{Br}\left[\left(-\psi_{r r}+\frac{1}{r+k} \psi_{r}\right) S_{r x}\right] \\
& +\mathrm{Du}\left[\phi_{r r}+\frac{1}{r+k} \phi_{r}\right]=0, \\
& \phi_{r r}+\frac{1}{r+k} \phi_{r}+\operatorname{Sc~Sr}\left[\theta_{r r}+\frac{1}{r+k} \theta_{r}\right]=0 \\
& S_{r x}=\frac{1}{1+\lambda_{1}}\left(-\psi_{r r}+\frac{1}{r+k} \psi_{r}\right),
\end{aligned}
$$

where $\theta$ and $\phi$ are the non-dimensionlized temperature and concentration profiles. Now, the dimensionless boundary conditions are:

$$
\begin{aligned}
& \psi_{r}=0, \quad \text { at } \quad r= \pm \eta, \\
& {\left[E_{1} \frac{\partial^{3}}{\partial x^{3}}+E_{2} \frac{\partial^{3}}{\partial x \partial t^{2}}+E_{3} \frac{\partial^{2}}{\partial t \partial x}\right] \eta} \\
& =\frac{1}{k(r+k)} \frac{\partial}{\partial r}\left\{(r+k)^{2} S_{r x}\right\}-\frac{\mathrm{Ha}^{2} \psi_{r}}{k(r+k)} \\
& \text { at } r= \pm \eta,
\end{aligned}
$$

$$
\begin{aligned}
& \theta=1, \quad \theta=0, \quad \text { at } \quad r= \pm \eta, \quad \text { and } \\
& \phi=1, \quad \phi=0, \quad \text { at } \quad r= \pm \eta, \\
& \eta(x)=[1+\gamma \sin 2 \pi(x-t)] .
\end{aligned}
$$

Results for planner channel can be obtained when curvature parameter $k$ is large, i.e. $k \rightarrow \infty$.

\section{Solution procedure}

The non-dimensionlized boundary value problem given in Eqs. (20)-(22) corresponding to boundary conditions (24)-(26) is calculated for stream function, $\psi$, temperature profile, $\theta$, and concentration profile, $\phi$. The numerical solution is presented utilizing the built-in shooting algorithm in Mathematica software.

\section{Physical interpretation}

The prime objective in this section is to scrutinize the outcome of involved physical variables on different flow quantities. Figure 2 (a)-(d) show the influences of pertinent parameters which are present in the momentum equation of the considered problem. Velocity profile has accelerated behavior for rising values of curvature parameter $k$ (see Figure 2(a)). The effects of the compliant wall parameters on velocity profile, $\nu_{2}$, are drawn in Figure 2(b). Also, disturbance is observed in the symmetry of velocity profile, $\nu_{2}$, about the center line of the channel, and symmetry retains its position for larger curvature parameter $k$. It is observed that larger values of $E_{1}, E_{2}$, and $E_{3}$ enhance the velocity. It is also important to know that an increase in $E_{1}$ and $E_{2}$ enhances velocity inside the channel, while it decreases when damping parameter, $E_{3}$, increases. Figure 2(c) explores that velocity profile, $\nu_{2}$, augments in nonNewtonian fluids $\left(\lambda_{1} \neq 0\right)$ when matched with the Newtonian liquid $\left(\lambda_{1}=0\right)$. The behavior of Hartman number on velocity profile, $\nu_{2}$, is plotted in Figure $2(\mathrm{~d})$. It reveals that the velocity decreases in larger radial magnetic field parameters, i.e. the magnetic force has a resistive role in the flow.

Figure 3(a)-(e) indicate the variation in temperature $\theta$, for the pertinent physical parameters. Figure 3(a) illustrates that the temperature decreases in the case of curvature parameter, $k$. Temperature increases in the case of different values of compliant wall parameters, $E_{1}, E_{2}$, and $E_{3}$ (see Figure $3(\mathrm{~b})$ ). It is observed that the temperature is enhanced inside the channel for larger wall elastance parameter, $E_{1}$, or mass characterizing parameter, $E_{2}$. Note that the temperature drops within the channel for greater damping parameter, $E_{3}$. Figure $3(\mathrm{c})$ depicts that temperature profile $\theta$ increases in different values of 


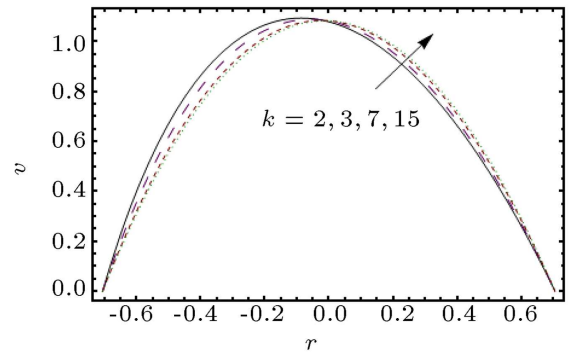

(a)

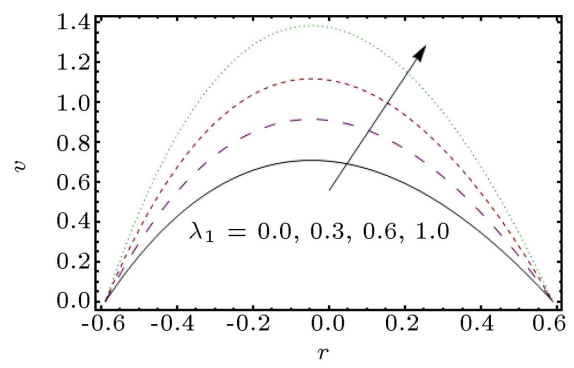

(c)

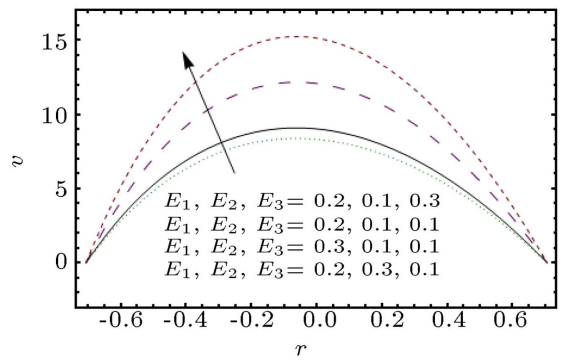

(b)

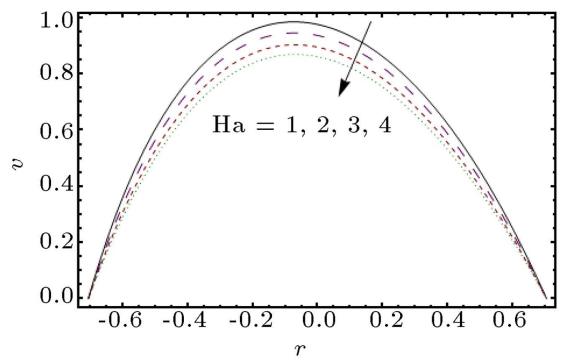

(d)

Figure 2. Variation in $\nu_{2}$ for different parameters when $\gamma=0.7, x=-0.2, t=0.2$ : (a) $\lambda_{1}=0.2, \mathrm{Ha}=1.0, E_{1}=0.2$, $E_{2}=0.1, E_{3}=0.1$, (b) $\lambda_{1}=0.2, \mathrm{Ha}=1.0, k=2.5$, (c) $k=2.5, \mathrm{Ha}=1.0, E_{1}=0.2, E_{2}=0.1, E_{3}=0.1$, and $(\mathrm{d}) \lambda_{1}=0.2$, $k=2.5, E_{1}=0.2, E_{2}=0.1, E_{3}=0.1$.

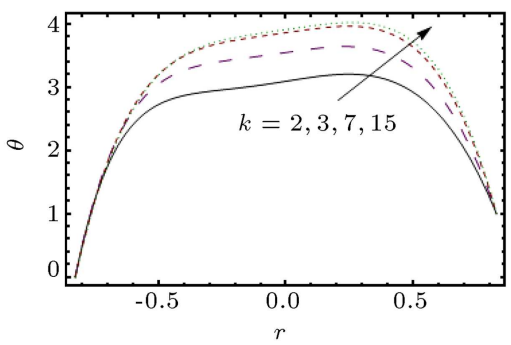

(a)

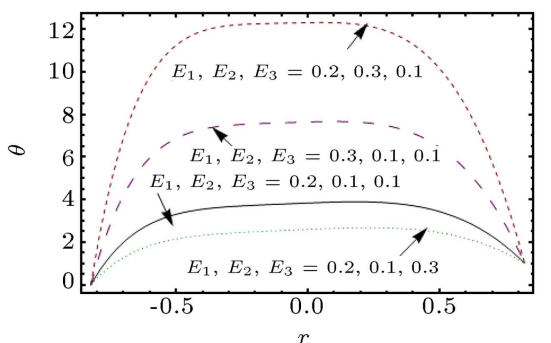

(b)

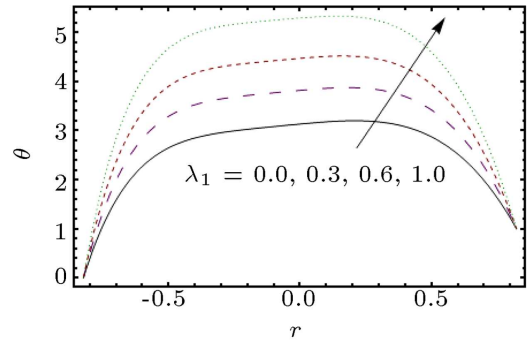

(c)

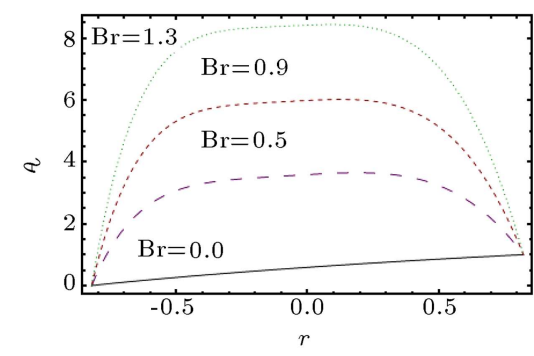

(d)

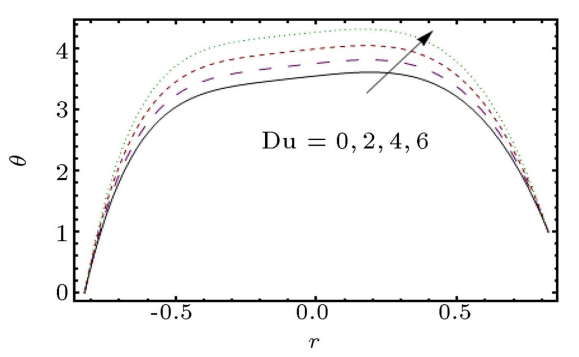

(e)

Figure 3. Variation in temperature $\theta$ for different sundry parameters when, $\gamma=0.7, x=-0.2, t=0.2, \operatorname{Pr}=2.0, \mathrm{Sr}=0.5$, $\mathrm{Sc}=0.5, \mathrm{Ha}=1.0:$ (a) $\lambda_{1}=0.2, \mathrm{Br}=0.5, E_{1}=0.2, E_{2}=0.1, E_{3}=0.1, \mathrm{Du}=0.5$, (b) $\lambda_{1}=0.2, \mathrm{Br}=0.5, k=2.5, \mathrm{Du}$ $=0.5$, (c) $k=2.5, \mathrm{Br}=0.5, E_{1}=0.2, E_{2}=0.1, E_{3}=0.1, \mathrm{Du}=0.5$, (d) $\lambda_{1}=0.2, k=2.5, E_{1}=0.2, E_{2}=0.1, E_{3}=0.1$, $\mathrm{Du}=0.5$, and $(\mathrm{e}) \lambda_{1}=0.2, \mathrm{Br}=0.5, k=2.5, E_{1}=0.2, E_{2}=0.1, E_{3}=0.1$.

Jeffrey fluid parameter $\left(\lambda_{1}\right.$, i.e. the relaxation factor is the only cause of increasing the temperature of fluid in the vicinity of the upper wall of the curved channel, Figure 3(d) shows that the temperature rises in terms of Brinkman number Br. Physically, it means that the increasing $\mathrm{Br}$ indicates larger amount of energy loss, i.e. maximum heat is produced because of its resistance against the shear stress in flow field, which enhances the temperature of the material. The temperature attains its maximum value towards the upper wall of the curved channel. It is also found that the temperature enhances upon increasing the values of Dufour number Du (see Figure 3(e)). It reveals that the temperature attains its maximum value at the center of the curved channel.

The effects of pertinent sundry variables on con- 
centration, $\phi$, can be seen through Figures 4(a)-(e). Concentration, $\phi$, shows the decreasing behavior by increasing curvature, $k$ (see Figure $4(\mathrm{a})$ ). Figure $4(\mathrm{~b})$ characterizes that the concentration profile decreases in non-Newtonian fluid when correlated with the Newtonian liquid. It means that the retardation time of the Jeffrey fluid parameter retards concentration, $\phi$, of the fluid near the lower wall of the curved channel. Figure 4(c) indicates fluid concentration, $\phi$, for particular values of elasticity parameters. Surprisingly, $\phi$, becomes negative in few values of parameters. It is realistic when nutrients are dispersed away from the blood vessels towards the nearby tissues. We have seen that $\phi$ reduces upon enhancing wall elastance, $E_{1}$, and wall mass characterizing, $E_{2}$. However, concentration, $\phi$, is an increasing quantity of $E_{3}$. Figure $4(\mathrm{~d})$ and (e) present that the concentration decreases in distinct values of Soret number, Sr, and Schmidt number, Sc.

Figure 5(a)-(e) indicate the fluctuation in heat transfer coefficient, $Z$. Obviously, the behavior of



(a)

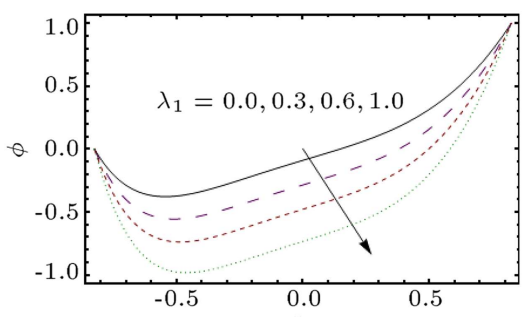

(b)



(c)



(d)

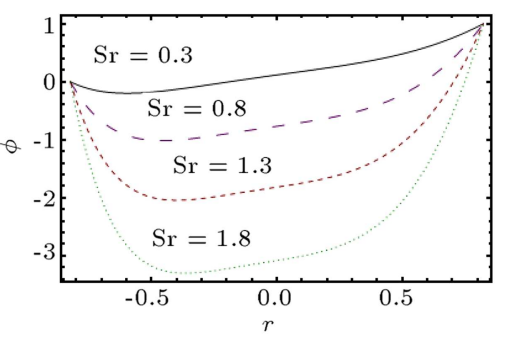

(e)

Figure 4. Variation in concentration $\phi$ for different pertinent parameters when $\gamma=0.7, x=-0.2, t=0.2, \mathrm{Pr}=2.0, \mathrm{Br}$ $=0.5, \mathrm{Du}=0.5, \mathrm{Ha}=1.0:$ (a) $\lambda_{1}=0.2, \mathrm{Sr}=0.5, E_{1}=0.2, E_{2}=0.1, E_{3}=0.1, \mathrm{Sc}=0.5$, (b) $\mathrm{Sr}=0.5, k=2.5, E_{1}=0.2$, $E_{2}=0.1, E_{3}=0.1, \mathrm{Sc}=0.5$, (c) $\lambda_{1}=0.2, k=2.5, \mathrm{Sr}=0.5, \mathrm{Sc}=0.5$, (d) $\lambda_{1}=0.2, k=2.5, E_{1}=0.2, E_{2}=0.1, E_{3}=0.1$, $\mathrm{Sc}=0.5$, and (e) $\lambda_{1}=0.2, \mathrm{Sr}=0.5, k=2.5, E_{1}=0.2, E_{2}=0.1, E_{3}=0.1$.

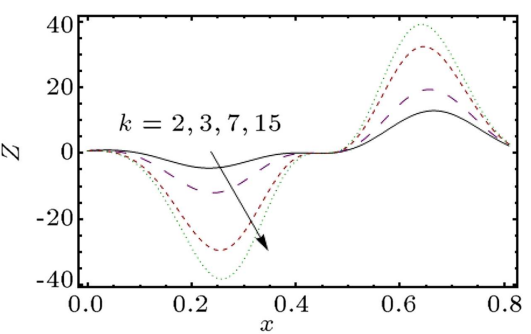

(a)



(b)

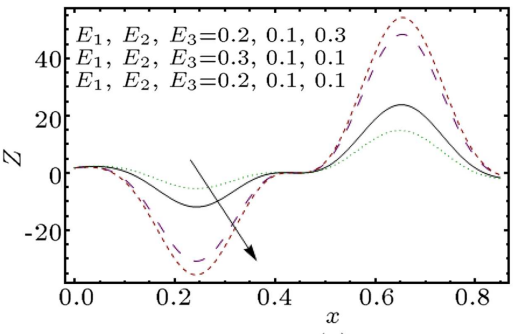

(c)



(d)

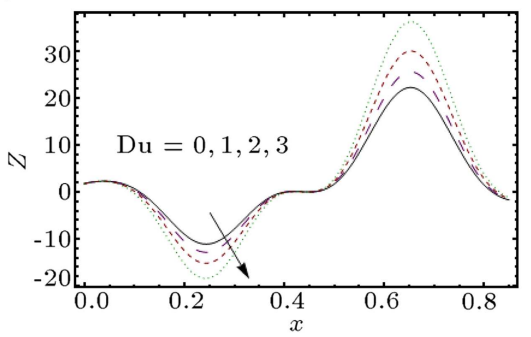

(e)

Figure 5. Variation in heat transfer coefficient $Z$ for different parameters when $\gamma=0.7, t=0.2, \operatorname{Pr}=2.0, \mathrm{Sr}=0.5, \mathrm{Sc}$ $=0.5, \mathrm{Ha}=1.0$ : (a) $\lambda_{1}=0.2, \mathrm{Br}=0.5, E_{1}=0.2, E_{2}=0.1, E_{3}=0.1, \mathrm{Du}=0.5$, (b) $\mathrm{Br}=0.5, k=2.5, \mathrm{Du}=0.5$, $E_{1}=0.2, E_{2}=0.1, E_{3}=0.1$, (c) $\lambda_{1}=0.2, k=2.5, \mathrm{Br}=0.5, \mathrm{Du}=0.5$, (d) $\lambda_{1}=0.2, k=2.5, E_{1}=0.2, E_{2}=0.1$, $E_{3}=0.1, \mathrm{Du}=0.5$, and $(\mathrm{e}) \lambda_{1}=0.2, \mathrm{Br}=0.5, k=2.5, E_{1}=0.2, E_{2}=0.1, E_{3}=0.1$. 
$Z$ is oscillatory. Figure 5(a) depicts that the heat transfer coefficient decays when curvature parameter $k$ enhances. Figure $5(\mathrm{~b})$ portrays that an increase in $\lambda_{1}$ causes a decrease in the coefficient of heat transfer. In Figure $5(\mathrm{c})$, it is observed that compliant wall parameters, $E_{1}, E_{2}$, and $E_{3}$, decrease, $Z$. Variation in $Z$ for larger viscous dissipation effects on (i.e., of Brinkman number) $\mathrm{Br}$ is depicted in Figure $5(\mathrm{~d})$. It is revealed that $Z$ decreases by Br. Figure 5(e) illustrates that $Z$ is also the decreasing function of Du number.

Figures 6-8 show the variation in streamlines for different physical parameters. Figure 6(a)-(c) are plotted for variation of curvature parameter, $k$, in stream function, $\psi$. It depicts that, in larger curvature parameter, $k$, reduces the size and circulation of trapped bolus in upper half of the channel, whereas the size of trapped bolus decreases and the number of circulation increases in lower half of channel. Figure 7 (a)-(c) reflect that the number of circulation and size of the trapped bolus reduce in case of larger radial magnetic parameter Ha. Figure $8(\mathrm{a})-(\mathrm{d})$ show that the size of trapped bolus decreases when compliant walls' parameters $E_{1}, E_{2}$, and $E_{3}$ increase.

\section{Concluding remarks}

Dufour and Soret effects on peristalsis subject to radial magnetic field are analyzed. The main findings are pointed out as follows:

- Velocity in inviscid fluid is more than the viscous material;

- The effects of $E_{1}$ and $E_{2}$ on temperature are opposite to that of $E_{3}$;

- There is an enhancement of temperature for Brinkman and Dufour numbers;

- As expected, the heat transfer coefficient has oscillatory characteristics;

- Circulation of trapped bolus decreases, whereas the size of the bolus increases in larger $\mathrm{Ha}$;



(a) $k=2$

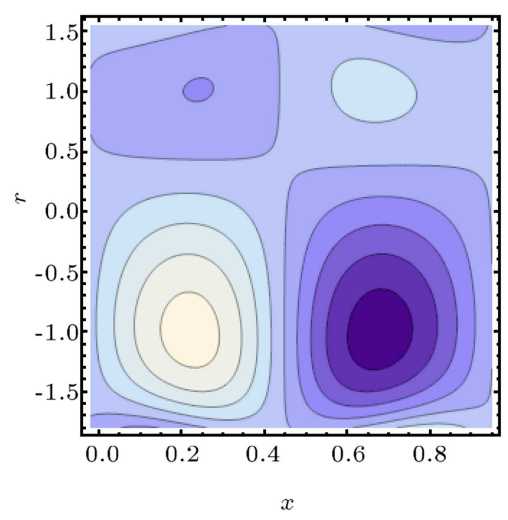

(b) $k=5$

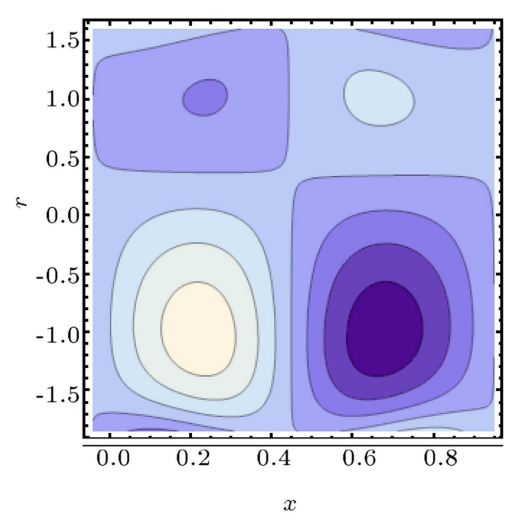

(c) $k=7$

Figure 6. Influence of curvature parameter $k$ on streamlines when $\gamma=0.7, t=0.2, \mathrm{Ha}=3.0, \lambda_{1}=0.2, E_{1}=0.2, E_{2}=0.1$, and $E_{3}=0.1$.

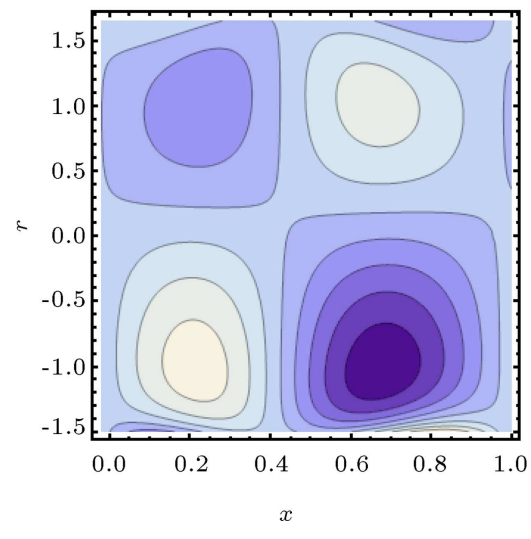

(a) $\mathrm{Ha}=1.0$

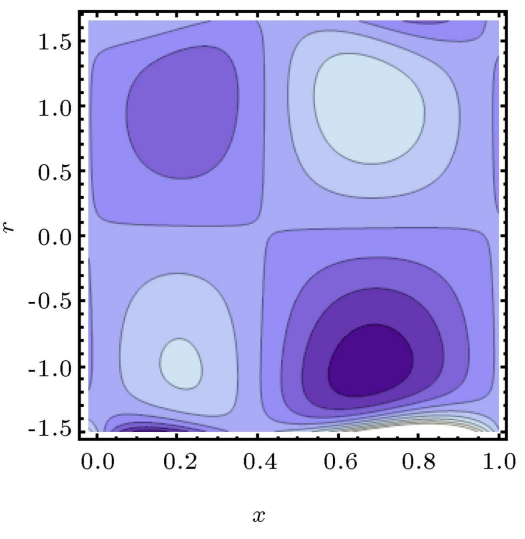

(b) $\mathrm{Ha}=2.0$

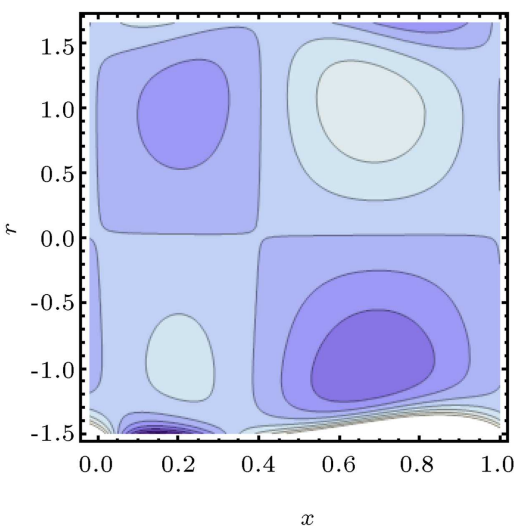

(c) $\mathrm{Ha}=3.0$

Figure 7. Influence of Hartman number, Ha, on streamlines when $\gamma=0.7, t=0.2, \lambda_{1}=0.2, k=2.5, E_{1}=0.2, E_{2}=0.1$, $E_{3}=0.1$. 


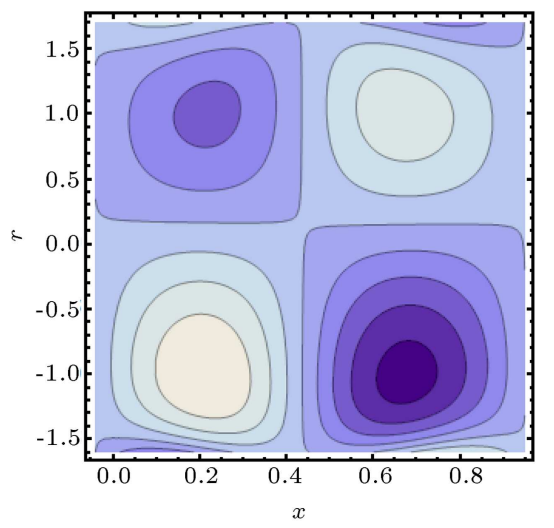

(a) $E_{1}=0.4, E_{2}=E_{3}=0.4$

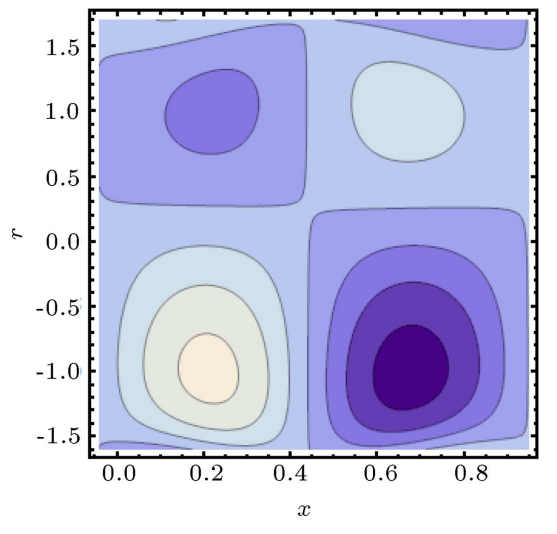

(c) $E_{1}=E_{2}=E_{3}=0.4$

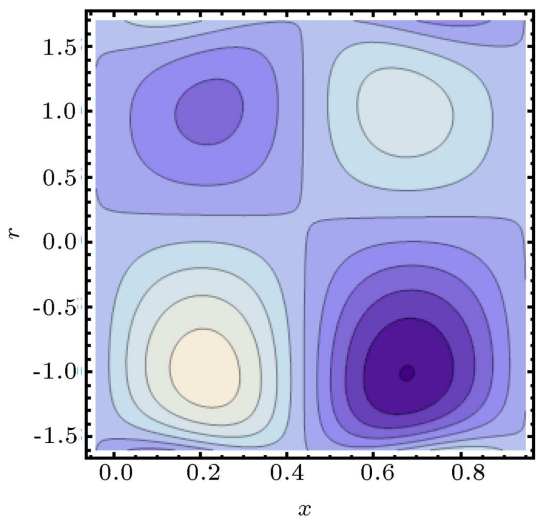

(b) $E_{1}=0.5, E_{2}=E_{3}=0.4$

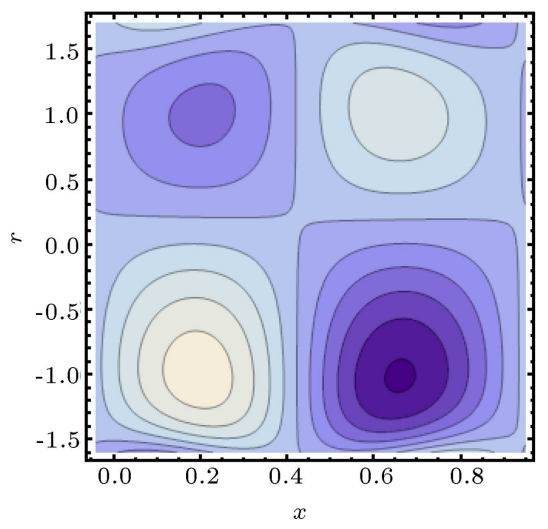

(d) $E_{1}=0.4, E_{2}=0.1, E_{3}=0.4$

Figure 8. Influence of compliant wall properties, $E_{1}, E_{2}, E_{3}$, on streamlines when $\gamma=0.7, t=0.2, \lambda_{1}=0.2, k=2.5$, $\mathrm{Ha}=3.0$.

- The effects of $E_{1}$ and $E_{3}$ on trapped bolus are quite opposite to that of $E_{2}$.

\section{References}

1. Latham, T.W. Fluid Motion in a Peristaltic Pump, MIT, Cambridge MA (1966).

2. Shapiro, A.H., Jaffrin, M.Y. and Weinberg, S.L. "Peristaltic pumping with long wavelength at low Reynolds number", J. Fluid Mech., 35, pp. 799-825 (1969).

3. Tripathi, D.A. "Mathematical model for swallowing of food bolus through the esophagus under the influence of heat transfer", Int. J. Therm. Sci., 51, pp. 91-101 (2012).

4. Mekheimer, Kh.S., Abd Elmaboud, Y. and Abdellateef, A.I. "Particulate suspension flow induced by sinusoidal peristaltic waves through eccentric cylinders: thread annular", Int. J. Biomath., 06, p. 1350026 (2013) .

5. Mustafa, M., Abbasbandy, S., Hina, S. and Hayat, T. "Numerical investigation on mixed convective peristaltic flow of fourth grade fluid with Dufour and Soret effects", J. Taiwan Inst. Chem. Eng., 45, pp. 308-316 (2014).
6. Mekheimer, Kh.S. and Abd elmaboud, Y. "Simultaneous effects of variable viscosity and thermal conductivity on peristaltic flow in a vertical asymmetric channel", Can. J. Phys., 92, pp. 1541-1555 (2014).

7. Gad, N.S. "Effects of hall currents on peristaltic transport with compliant walls", Appl. Math. Comput., 235, pp. 546-554 (2014).

8. Sinha , A., Shit, G.C. and Ranjit, N.K. "Peristaltic transport of MHD flow and heat transfer in an asymmetric channel: Effects of variable viscosity, velocityslip and temperature jump, Alexandria", Eng. J., 54, pp. 691-704 (2015).

9. Ellahi, R. Bhatti, M.M. Riaz, A. and Sheikoleslami, M. "The effect of magnetohydrodynamics on peristaltic flow of Jeffrey fluid in a rectangular duct through a porus medium", J. Porus Media., 17, pp. 1-20 (2014).

10. Awais, M., Farooq, S., Yasmin, H., Hayat, T. and Alsaedi, A. "Convective heat transfer analysis for MHD peristaltic flow of Jeffrey fluid in an asymmetric channel", Int. J. Biomath., 7, 1450023 (2014).

11. Kothandapani, M. and Srinivas, S. "Peristaltic transport of Jeffrey fluid under the effect of magnetic field in an asymmetric channel", Int. J. non-Linear Mech., 43, pp. 915-924 (2012). 
12. Hayat, T., Bibi, A., Yasmin, H. and Ahmad, B. "Simultaneous effects of Hall current and homogeneous/heterogeneous reactions on peristalsis", J. Taiwan Inst. Chem. Eng., 58, pp. 28-38 (2016).

13. Asghar, S., Minhas, T. and Ali, A. "Existence of a Hartmann layer in the peristalsis of Sisko fluid", Chin. Phys. B., 23, pp. 054702-054707 (2014).

14. Hina, S., Mustafa, M., Hayat, T. and Alotaibi, N.D. "On peristaltic motion of pseudoplastic fluid in a curved channel with heat/mass transfer an wall properties", Appl. Math Comput., 263, pp. 378-391 (2015).

15. Hayat, T., Hina, S., Hendi, A.A. and Asghar, S. "Effects of wall properties on the peristaltic flow of a third grade fluid in a curved channel with heat and mass transfer", Int. J. Heat Mass Transfer., 54, pp. 5126-5136 (2012).

16. Ali, N., Sajid, M., Abbas, Z. and Javed, T. "NonNewtonian fluid flow induced by peristaltic waves in a curved channel", Eur. J. Mech. B/Fluids, 29, pp. 387-394 (2010).

17. Shehzad, S.A., Abbasi, F.M., Hayat, T., Alsaadi, F. and Mousae, G. "Peristalsis in a curved channel with slip condition and radial magnetic field", Int. J. Heat Mass Transfer., 91, pp. 562-569 (2015).

18. Vajravelua, K., Sreenadh, S. and Saravana, R. "Combined influence of velocity slip, temperature and concentration jump conditions on MHD peristaltic transport of a Carreau fluid in a non-uniform channel", Appl. Math. Comput., 225, pp. 656-676 (2013).

19. Hina, S., Mustafa, M. and Hayat, T. "On the exact solution for peristaltic flow of Couple-stress fluid with wall properties, "Bulg. Chem. Commun"., 47, pp. 3037 (2015).

20. Abd-Alla, A.M. and Abo-Dahab, S.M. "Magnetic field and rotation effects on peristaltic transport of a Jeffrey fluid in an asymmetric channel", J. Mag. Mag. Mater., 374, pp. 680-689 (2015).

21. Hayat, T., Bibi, S., Alsaadi, F. and Rafiq, M., "Peristaltic transport of Prandtl-Eyring liquid in a convectively heated curved channel", PLoS One., 11, e0156995 (2016).

22. Ellahi, R., Bhatti, M.M. and Pop, I. "Effects of hall and ion slip on MHD peristaltic flow of Jeffrey fluid in a non-uniform rectangular duct", Int. J. Numer. Methods Heat Fluid Flow., 26, pp. 1802-1820 (2016).

23. Hayat, T., Farooq, S., and Alsaedi, A. "MHD peristaltic flow in a curved channel with convective condition", J. Mech, 33, pp. 483-499 (2017).
24. Khan, A.A., Muhammad, S., Ellahi, R. and Zia, Q.M. Z. "Bionic study of variable viscosity on MHD peristaltic flow of pseudoplastic fluid in an asymmetric channel", J. Mag., 21, pp. 273-280 (2016).

25. Ellahi, R. and Hussain, F. "Simultaneous effects of MHD and partial slip on peristaltic flow of Jeffery fluid in a rectangular duct", J. Mag. Mag. Mater., 393, pp. 284-292 (2015)

26. Bhatti, M.M., Zeeshan, A. and Ellahi, R. "Endoscope analysis on peristaltic blood flow of Sisko fluid with titanium magneto-nanoparticles", Computers Bio. Med., 78, pp. 29-41 (2016).

27. Ellahi, R., Rahman, S.U., Nadeem, S. and Vafai, K. "The blood flow of Prandtl fluid through a tapered stenosed arteries in permeable walls with magnetic field", Communi. Theor. Phy., 63, pp. 353-358 (2015).

28. Awais, M., Hayat, T., Nawaz, M. and Alsaedi, A. "Newtonian heating, thermal-diffusion and diffusionthermo effects in an axisymmetric flow of a Jeffery fluid over a stretching surface", Braz. J. Chem. Eng., 32, pp. 555-561 (2015).

29. Hayat, T., Farooq, S., Ahmad, B. and Alsaedi, A. "Effectiveness of entropy generation and energy transfer on peristaltic flow of Jeffrey material with Darcy resistance", Int. J. Heat Mass Transfer., 106, pp. 244252 (2017).

30. Ali, N. Sajid, M. and Hayat, T. "Long wavelength flow analysis in a curved channel", Zeitschrift für Naturforschung A. 65, pp. 191-196 (2010).

31. Srivastava, L.M. and Srivastava, V.P. "Peristaltic transport of a power law fluid: Applications to the ductus efferentes of the reproductive tract", Rheol. Acta., 27, pp. 428-433 (1988).

32. Lew, S.H., Fung, Y.C. and Lowenstein, C.B. "Peristaltic carrying and mixing of chyme", J. Biomech., 4, pp. 297-315 (1971).

\section{Biographies}

Tasawar Hayat received his $\mathrm{PhD}$ degree in Applied Mathematics from Quaid-i-Azam University, Islamabad, Pakistan, in 1999. Since then, he has been in teaching and research at Quaid-i-Azam University, Islamabad, Pakistan, where he joined as a lecturer in Department of Mathematics, 1998, and has been promoted to a Professor. Besides, he has been appointed as Distinguished National Professor by the Higher Commission of Pakistan. He has published extensively in Newtonian and non-Newtonian fluid mechanics. He has received a number of national and international awards, and is well known internationally.

Shahid Farooq received his $\mathrm{PhD}$ degree in Applied Mathematics from Quaid-i-Azam University, Islamabad, Pakistan, in 2018. His works mainly address the peristaltic flows of Newtonian and non-Newtonian 
materials. He published interesting articles in international Journals of high repute.

Bashir Ahmad received his PhD in 1995 from Quaidi-Azam University, Islamabad, Pakistan. His research interest includes nonlinear ordinary/integro-differential equations, fractional differential equations, stability analysis, and wave motion. He has published exten- sively. He has been declared as the best researcher of King Abdulaziz University in 2009. At present, he has 18 h-index. He is the reviewer of several international journals. He is the assistant managing editor of Bulletin Mathematical Sciences and associate editor of Advances in Difference Equations. He has already completed 11 research projects successfully. He also has supervised several research students. 